\title{
Sistemas biomiméticos de captura y filtración de agua pluvial para casas particulares y unidades habitacionales
}

\author{
Jesús Emanuel Juárez Rojoa, Miguel Ángel Cervantes Santiagoa ${ }^{a}$, Tomás A. Fregoso Aguilara ${ }^{a}$, Perla \\ Inés Badillo Lagunes ${ }^{\mathrm{a}}$, Jorge A. Mendoza Pérez ${ }^{\mathrm{a} *}$, Sergio Odín Flores Valle ${ }^{\mathrm{b}}$ y Omar Ríos Bernÿ ${ }^{\mathrm{b}}$ \\ ${ }^{a}$ Escuela Nacional de Ciencias Biológicas-IPN, Fax: 57296000 Ext 52301. 52312 \& 52336. Wilfrido Massieu S/N., Unidad Adolfo \\ López Mateos, Colonia Nueva Industrial Vallejo, Gustavo A. Madero, 07738, México D.F., México. \\ ${ }^{b}$ Escuela Superior de Ingeniería Química e Industria Extractiva- IPN., Unidad Profesional Adolfo López Mateos, Colonia Lindavista, \\ Código Postal 07738, Delegación Gustavo A. Madero, Ciudad de México, Distrito Federal, México. \\ *E-mail: jorgemendozaperez@yahoo.com
}

Recibido 13 de noviembre de 2012, Aceptado 7 de diciembre de 2012

\section{Resumen}

Hoy en día es una necesidad urgente con carácter social, de salud humana, ambiental e incluso política, asegurar el abastecimiento de agua a las ciudades y poblados de nuestro país. En el caso del Distrito Federal, esta ciudad cuenta con una geografía característica que permite cada año un aporte de agua de lluvia de casi 780 millones de $\mathrm{m}^{3}$ pero solo cerca del $13 \%$ de este volumen, se aprovecha. La naturaleza da ejemplos de cómo pueden ser diseñados los sistemas para capturar agua pluvial. Al proceso de estudiar las soluciones que la propia naturaleza ha adoptado se le conoce como biomimetismo y la biomimética es la tecnología que imita a la naturaleza. El objetivo de este trabajo fue copiar principios simples de la naturaleza para diseñar un sistema biomimético de captura y filtración de agua pluvial para casas particulares y unidades habitacionales. Para el diseño del captador biomimético se trabajó con materiales ligeros que no absorben agua sino que la conducen con rapidez a depósitos, debido a que se favorece el fenómeno de cohesión. De los estudios se obtuvo la impermeabilidad de los materiales, su resistencia al flujo y se calcularon los coeficientes de cohesión alcanzados, lo que indica que tanto la moléculas de agua se agrupan entre si y así se evita que se humedezcan los materiales captadores. Estos datos permitieron el diseño final de un primer prototipo.

Palabras clave: Pluvial, Captura, Filtración, Sustentable, Biomimético.

\section{Abstract}

Today there is an urgent need with a compromise of social kind as also with human health, environmental and even of political profile to ensure water supply to cities and towns of our country. In the case of Mexico City, this city has a geographical feature that allows an annual rainwater supply nearly 780 million $\mathrm{m}^{3}$ but only about $13 \%$ of this volume is used. Nature gives examples of how systems can be designed to capture rainwater. The process of studying the solutions that nature has adopted is known as biomimicry and biomimetics is the technology that mimics nature. The aim of this study was to copy simple principles of nature to design biomimetic systems which can be used to capture and filter rainwater for private homes and residential units. To design the biomimetic capture system several assays were performed with high resistance lightweight materials that do not absorb water but they lead the rainwater rapidly to the deposits, because the material coating favors the phenomenon of cohesion. From the performed studies was obtained the impermeability of the materials, their flow resistance and the cohesion coefficients also were calculated, thus indicating that the water molecules are grouped together and this prevents the capturing materials get moistened. The achieved data allowed the final design of a first prototype.

Keywords: Storm, Capture, Filtration, Sustainable, Biomimetic.

\section{Introducción}

Inevitablemente al hablar de captación de agua pluvial nos tenemos que remitir al ciclo hidrológico, de este tomamos la parte de la precipitación y filtración, que es el método de depuración de agua en la naturaleza, de esta manera al captar y filtrar el agua se está tomando como referencia el modelo de la Figura 1.

En la captación de agua, la parte más importante en cuanto a consumo humano se refiere, es la filtración del agua de lluvia ya que es en este punto donde se llevará al agua a un tratamiento para eliminar todos los contaminantes que fueron arrastrados por esta al precipitar. Los contaminantes presentes en la atmósfera del valle de México tales como plomo, dióxido de azufre de óxidos de nitrógeno y otros contaminantes que están presentes y que son traídos por arrastre. Es por eso que la filtración se vuelve un reto en esta parte de la ciudad, para que el agua no sea solamente utilizada para servicios sino también para el consumo humano. Entonces, el modelo principal ocupado en todos los sistemas de uso de agua de lluvia, son:

a) Captación de agua

b) Filtración de agua

La Captación de Agua Pluvial (CAP) es el término utilizado tradicionalmente para la 
recolección de agua de lluvia [1] en países húmedos $\mathrm{y}$ áridos, en contextos de pobreza y de riqueza, la captación de agua de lluvia es una fuente importante para el abastecimiento de agua en los hogares, la producción agrícola y el mantenimiento de espacios verdes y ecosistemas estratégicos para la vida urbana [2]. La planificación y la gestión del agua de lluvia pueden también reducir riesgos, prevenir daños a la salud y mitigar desastres. Los beneficios del uso de la lluvia van más allá del uso para consumo humano y animal. Nutre la conservación de bosques y humedales y disminuye la presión sobre acuíferos y proyectos de construcción de nuevas presas para el abastecimiento urbano [1].

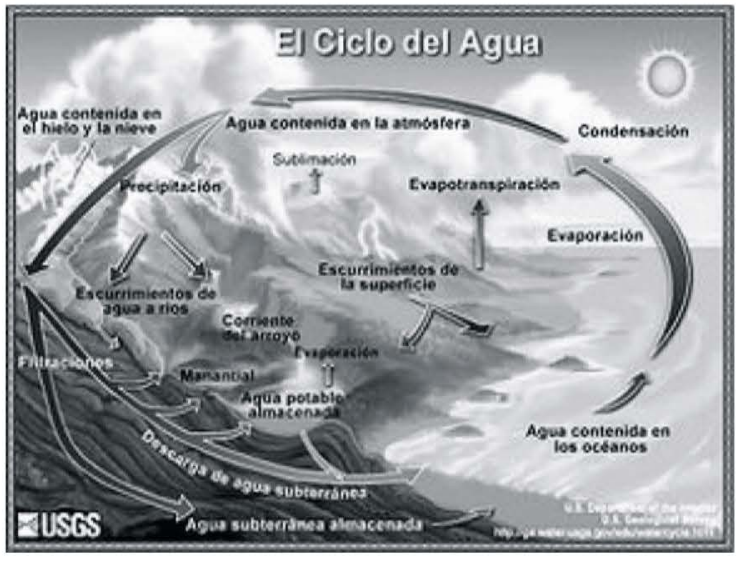

Figura 1. El ciclo del agua (USGS)

Los usos del agua de lluvia en la actualidad son:

-Domésticos y productivos

-Sanitarios y de higiene

-Protección de cuencas

-Soberanía alimentaria

- Conservación de suelos

- Recarga de acuíferos

-Preservación de ecosistemas estratégicos

-Prevención de desastres y estrategias de reconstrucción después de los desastres

La necesidad de una fuente alterna para el abastecimiento de agua en la ciudad se ha vuelto latente al ver la inminente escasez que le espera si no se cambia el sistema de abastecimiento, en este punto, queda comprobado que los grandes sistemas de red no son suficientes para el abastecimiento de la ciudad por lo que el uso de alternativas locales se presenta como una mejor opción para mejorar el abastecimiento de agua en el Distrito Federal.

\subsection{Filtración de agua}

La filtración es el proceso de retiro de sólidos suspendidos del agua mediante el paso del agua a través de una tela permeable o una cama de materiales porosos. El agua subterránea es filtrada naturalmente a medida que fluye a través de las capas porosas de la tierra. Algunos contaminantes plantean una amenaza a la salud humana y la filtración es uno de los más antiguos y simples métodos de retirarlos. Los métodos de filtración incluyen filtración lenta y rápida de arena, filtración de tierra diatómica, filtración directa, filtración de empaque, filtración de membrana y filtración de cartucho (Figura 2)

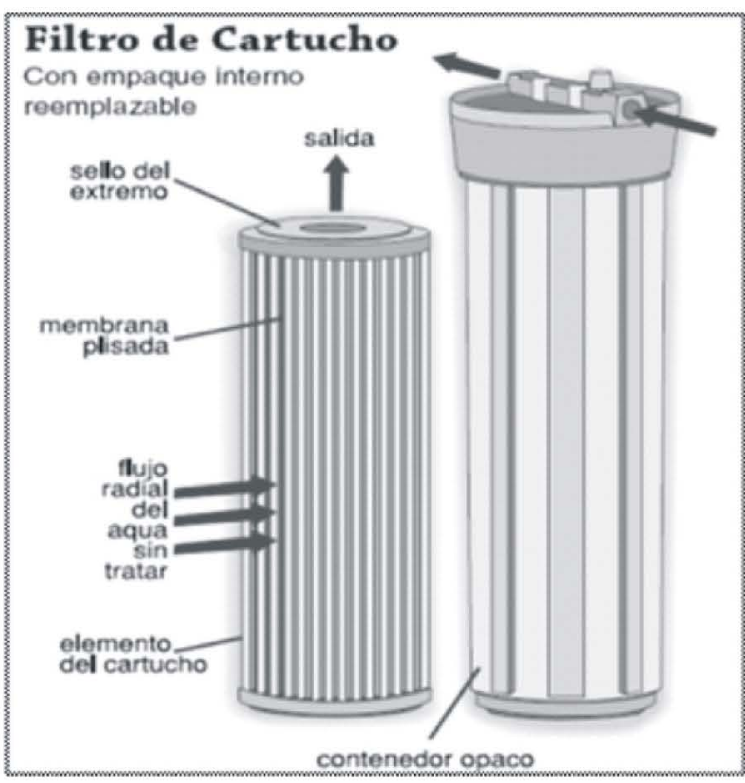

Figura 2. Filtro de cartucho (NESC West Virginia University)

La filtración es usualmente una combinación de procesos físicos y químicos. La filtración mecánica retira algunas partículas atrapándolas entre los granos del medio del filtro (como arena). La adhesión es un proceso igualmente importante por el cual las partículas suspendidas se pegan a la superficie de los granos del filtro o del material previamente depositado. Los procesos biológicos también son importantes en los filtros lentos de arena. Estos filtros forman una capa filtrante que contiene microorganismos que atrapan y destruyen algas, bacterias y otras materias orgánicas antes que el agua alcance el mismo medio del filtro [3]. Si bien el tratamiento que el agua pluvial necesita puede solucionarse con un filtro como el anteriormente citado, no es la única opción disponible para su tratamiento. Pero el modelo tomado por la naturaleza nos ayuda a tratar el agua pluvial, ahora solo necesitamos saber cuánta agua podemos captar. 


\subsection{Balance hidrológico del valle de México}

El valle de México tiene unas precipitaciones anuales de 700 milímetros, aunque las pérdidas por evaporación pueden llegar a ser del $70 \%$. Estas se concentran en los meses de junio a septiembre/octubre y durante el resto del año son escasas o nulas [4]. En la actualidad, apenas hay ríos permanentes; por lo tanto, las aguas subterráneas son el principal recurso hídrico del valle. El balance hidrológico representa la situación actual del agua y su perspectiva a futuro. A continuación se presentan los principales parámetros que intervienen en los balances hidráulicos de la Región XIII para un ciclo anual. Como puede observarse en la Tabla 1, en la cuenca del Valle de México los acuíferos se encuentran en una condición de sobreexplotación, por lo tanto no existe posibilidad de incrementar las extracciones, por el contrario, se deben de reducir las existentes a fin de obtener el equilibrio de los acuíferos de la subregión. En la cuenca del Río Tula existe una reducida disponibilidad de 40 millones $\mathrm{m}^{3} /$ año, pero en caso de incrementar la explotación de estos acuíferos, ésta deberá de ser planeada y controlada para no provocar la misma situación de sobreexplotación de la cuenca del Valle de México, además de que debido a la contaminación de estos acuíferos, para la utilización de esta fuente será necesario el control y tratamiento de las aguas residuales de la ZMCM, así como de la potabilización de las aguas de estos acuíferos [5]. La necesidad de una fuente alterna para el abastecimiento de agua en la ciudad se ha vuelto latente al ver la inminente escases que le espera si no se cambia el sistema de abastecimiento, en este punto, queda comprobado que los grandes sistemas de red no son suficientes para el abastecimiento de la ciudad por lo que el uso de alternativas locales se presenta como una mejor opción para mejorar el abastecimiento de agua en el Distrito Federal.

Tabla 1. Balance hidrológico del valle de México (Volúmenes en millones de $\mathrm{m}^{3}$, Conagua, 2012).

\begin{tabular}{cccccccc}
\hline Región Lluvia Evap. Infilt. & $\begin{array}{c}\text { Escorr. } \\
\text { Virgen }\end{array}$ & $\begin{array}{c}\text { Extracc. } \\
\text { de agua } \\
\text { Subt. }\end{array}$ & Balance \\
\hline $\begin{array}{c}\text { Valle } \\
\text { de }\end{array}$ & 6,646 & 5,377 & 689 & 580 & 1,584 & -895 \\
$\begin{array}{c}\text { México } \\
\text { Tula }\end{array}$ & 3,510 & 1,947 & 336 & 1,227 & 296 & 40 \\
\hline
\end{tabular}

En el balance presentado se consideran las pérdidas de agua de lluvia por evaporación (Evap), infiltración (Infilt.), escorrentías vírgenes (Escorr Virgen) y se compara en el balance, la diferencia entre el agua de lluvia infiltrada (Infilt.) como fuente de recarga con respecto a la extracción de agua subterránea (Extracc. de agua Subt.) dando un déficit de -895 millones de $\mathrm{m}^{3}$ para el Valle de México y apenas una volumen a favor de 40 millones de $\mathrm{m}^{3}$ para la región de Tula. En la Figura 3 se presentan los procesos que participan en el balance hídrico del Distrito Federal.

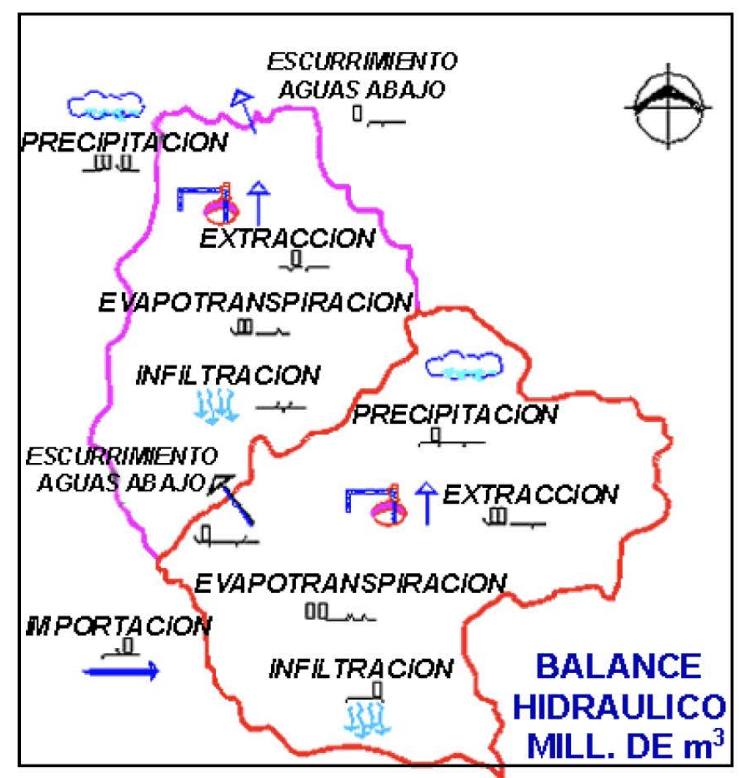

Figura 3. Balance hidrológico del Distrito Federal en millones de $\mathrm{m}^{3}$ (Conagua, 2012)

\subsection{La problemática del agua}

El agua es esencial para la sostenibilidad urbana. Tan solo en las últimas décadas la población en la ciudad México aumento a una tasa del $3.1 \%$ y en el último censo registrado alcanzo los $8,851.080$ de habitantes situando al Distrito Federal en el segundo lugar a nivel nacional por su número de habitantes [6]. Por lo que se requiere de políticas que hagan que esta ciudad sea ambientalmente sostenible. Desafortunadamente, la realidad muestra que la ciudad de México es cada vez menos sostenible en todos los aspectos, pero en especial en el abastecimiento de agua, y es ahora cuando las opciones descentralizadas de suministro, como lo es la captación de agua lluvia, requieren ser seriamente consideradas como alternativas viables para reducir la presión sobre la demanda creciente de agua de la red.

En México, el Centro Internacional para la Formación y Demostración sobre el Aprovechamiento del Agua Lluvia (CIDECALLI), 
del Colegio de Posgraduados, provee información valiosa para demostrar los beneficios comunitarios de sistemas de captación del agua pluvial, en particular en zonas marginales y en comunidades de bajos recursos. A pesar de muchos años de experimentación y ejecución de proyectos rurales, subsisten dificultades de incluir la lluvia en la agenda política de los Estados. Los estudios relacionados están enfocados en las características físicas, químicas y biológicas del agua de lluvia [7] mostrando la calidad que tiene y cantidad que caen en el valle México.

Algunos otros estudios del CIIEMAD están enfocados en la captación de agua pluvial, sin embargo, está dirigida a la recarga de mantos acuíferos como viene referenciado en la tesis "Aporte de agua pluvial al acuífero de la zona metropolitana del valle de México, captada en azoteas de edificios ${ }^{23}$ [8]. Por otra parte la CONAFOR menciona la importancia de la recolección y almacenamiento de agua de lluvias para el uso en actividades productivas con tratamientos primarios de filtros de arena [9], sin embargo no se contempla su uso para el consumo humano. En ninguno de los trabajos realizados anteriormente resalta la necesidad que tiene el Distrito Federal de contar con equipos de abastecimiento de agua que no modifiquen las estructuras de la ciudad o que comprometan un espacio público. Por lo que es necesario crear nuevas tecnologías en cuanto a la captación de agua de lluvia se refiere. De acuerdo con el último reporte de la Organización para la Cooperación y el Desarrollo (OCDE), de no cambiarse las políticas de manejo y cobro del agua, México enfrentará niveles de escasez de medios a severos en las próximas cuatro décadas [10]. Esta es una de las razones por la cuales las personas en el Distrito Federal buscan maneras alternas de suministro de agua, tal como el uso de pipas, e incluso agua almacenada en ríos cercanos a la comunidad, que comúnmente son canales de infección. No solamente es la falta de agua, sino la pobre calidad con la que llega a algunos hogares, esto no debido a la fuente, sino a la falta de mantenimiento de la infraestructura de suministro a delegaciones como Iztapalapa donde se sufre de manera continua de la escasez creciente en la ciudad.Los principales problemas son la sobreexplotación del acuífero, la mínima captación de agua de lluvia y el desperdicio del líquido que hay en las redes [11]. Analizando las razones anteriores, una de las formas más atractivas que tiene el Distrito Federal para el suministro de agua es la captación de agua pluvial, ya que es económicamente viable para la mayoría de los habitantes y proporciona el valor agregado de ser amigable con el ambiente.

\section{Parte Experimental}

Se realizó a través del servicio meteorológico nacional un balance hidrológico del Distrito Federal, del año 1978 al 2008 para tener datos confiables de las delegaciones donde más llueve y se obtuvo lo siguiente:

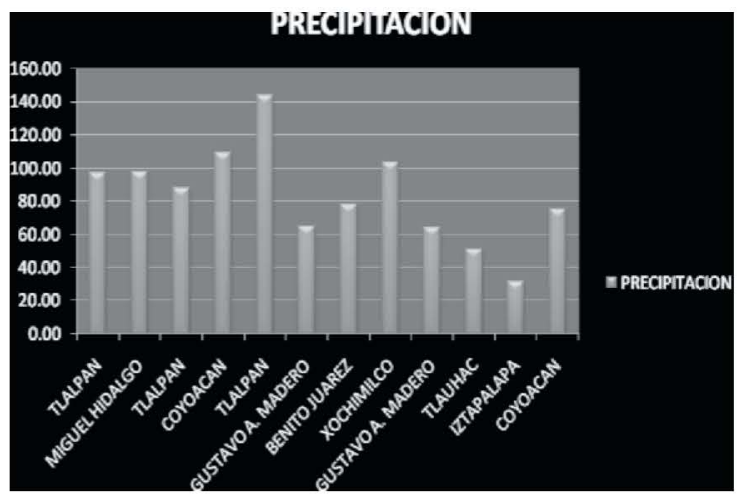

Figura 4. Precipitación promedio en el Distrito Federal desde 1978 hasta 2008 (delegaciones y secciones con mayor régimen pluviométrico)

Pero como el clima ha cambiado en los últimos 10 años el estudio se acotó a los datos de 2000 a 2008 obteniendo lo siguiente:

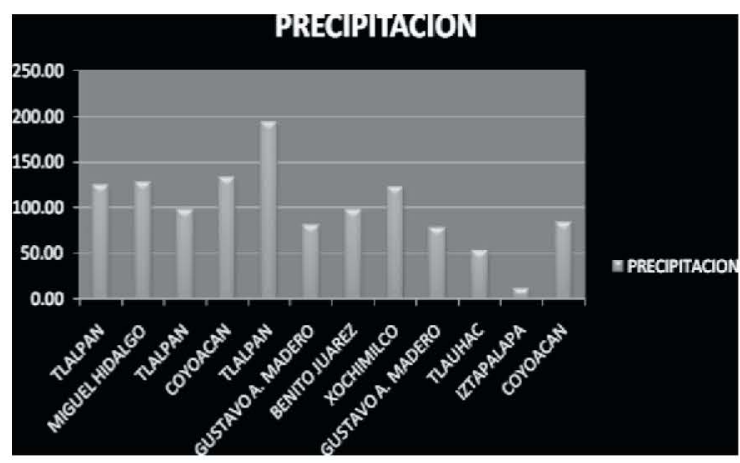

Figura 5. Precipitación promedio en el Distrito Federal desde el año 2000 hasta 2010 (delegaciones y secciones con mayor régimen pluviométrico)

Por lo que tras el análisis de ambos periodos, se concluyó que se colocaran los captadores de lluvia en las delegaciones Tlalpan sección III, Coyoacán sección I, Miguel Hidalgo y Xochimilco que son donde se presentan las mayores precipitaciones pluviales. Los datos de Xochimilco no se presentan en este documento por la riqueza de resultados 
conseguidos para el estudio de esa zona.

A continuación se realizó la construcción de pluviómetros, lo que tuvo lugar en el laboratorio de Tecnologías limpias de la ENCB-IPN.

Datos generales de dos pluviómetros.

Área del cono captador: $0.52 \mathrm{~m}^{2}$

Volumen cono captador: $37 \mathrm{~L}$

Área del círculo del cono:

Pluviómetros: Clave Azul

Tiempo de retención: $1 \mathrm{~min}$

Presión manométrica: $23 \mathrm{lb} / \mathrm{in}^{2}$

Flujo: $120 \mathrm{~L} / \mathrm{m}$---- Área de captación: $2 \mathrm{~m}^{2}$

Volumen capturado: $3.52 \mathrm{~L} / \mathrm{m}$---- Área: 6440 $\mathrm{cm}^{2}$

Nivel: 1

Condiciones ideales: $5.76 \mathrm{~L} / \mathrm{m}$

Equivalente de volumen en el recipiente: $1 \mathrm{~cm}$ $0.64 \mathrm{~L}$

Pluviómetros: Clave Naranja

Tiempo: $1 \mathrm{~min}$

Presión: $23 \mathrm{lb} / \mathrm{in}^{2}$

Flujo: $120 \mathrm{~L} / \mathrm{m}$---- Área: $2 \mathrm{~m}^{2}$

Volumen capturado: $3.0 \mathrm{~L} / \mathrm{m}$---- Área: 8140 $\mathrm{cm}^{2}$

Nivel: 1

Equivalente de volumen en el recipiente: $1 \mathrm{~cm}$ $0.87 \mathrm{~L}$

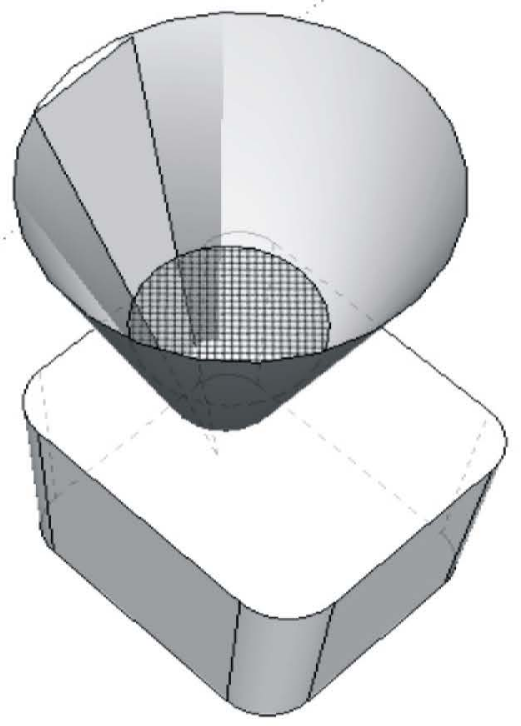

Figura 6. Pluviómetros construidos para medir regímenes pluviales en cuatro delegaciones del Distrito Federal.

Estos pluviómetros fueron colocados en casas privadas $\mathrm{y}$ edificios públicos en las delegaciones mencionadas y el agua pluvial captada era medida después de cada lluvia por los mismos habitantes de las casas o personal de servicio de los edificios públicos. Los datos se obtenían semanalmente al igual que muestras del agua colectada para su análisis según la NOM-127- SSA1-1994 de \Salud ambiental, agua para uso y consumo humanolímites permisibles de calidad y tratamientos a que debe someterse el agua para su potabilización $\square$

Con los resultados obtenidos se inició el diseño de los captadores biomiméticos denominados telas de araña. Se seleccionaron una serie de telas y recubrimientos de polímeros hidrófobos de silicón, ceras naturales y ceras sintéticas que fueron probados al tiempo que se obtenían los valores de humectabilidad mediante un Equipo "Contact Angle $^{22} \mathrm{KSV}$, modelo CAM 200, empleado para la medición de ángulos de contacto. Los recubrimientos se realizaron mediante deposición en caliente sobre las telas y dejar secar $30^{\circ} \mathrm{C}$ en estufa durante una noche ( 8 horas). También se obtuvo la tensión superficial del sólido mediante las normas ASTM D7490 para medir tensión superficial de revestimientos y la D7334 para humectabilidad de los mismos. La ecuación empleada para el cálculo de las energías superficiales de la fase sólido/liquida a partir de los ángulos de contacto y la tensión superficial del agua fue la del modelo de Young-Dupre:

$$
W_{S L}=\gamma_{L V}(1+\operatorname{Cos} \theta)
$$

El coeficiente de cohesión (CC) del agua sobre la superficie del sólido (tela) se calcula a partir del ángulo de contacto obtenido mediante la siguiente ecuación:

$$
C C=\frac{\emptyset}{180^{\circ}}
$$

Donde se considera que el coeficiente de cohesión más alto (1.00) se obtiene cuando el ángulo de contacto es de $180^{\circ}$, esto indica que no hay humectación o mojabilidad por parte de la gota sobre la superficie de la tela. Como material de referencia se utiliza una superficie de PTFE (teflón) con agua bidestilada cuyo ángulo de contacto es de $115^{\circ}$

Para determinar la capacidad de captura de agua y los cambios en el coeficiente de cohesión del agua sobre la superficie del material según envejece o se desgasta, se realizaron las siguientes pruebas:

1. Pruebas de Infiltración estática de mallas tratadas. Es el volumen de agua retenida (estática) que moja la tela y se filtra a través de un $\mathrm{m}^{2}$ de 
malla recubierta con un material hidrófobo por unidad de tiempo. La prueba se realiza reteniendo 1 $\mathrm{L}$ de agua en un área determinada de malla $\left(0.5 \mathrm{~m}^{2}\right)$ y se mide el tiempo que tarda en infiltrarse en su totalidad, la prueba tiene una duración máxima de 240 minutos (4 horas). Unidades $\mathrm{L} / \mathrm{m}^{2} \cdot \mathrm{min}$

2. Pruebas Infiltración dinámica de mallas tratadas. Es el volumen de un flujo de agua por $\mathrm{m}^{2} \mathrm{y}$ por unidad de tiempo, que no es retenida por las mallas recubiertas con material hidrófobo. La prueba se realiza con un flujo de $0.1 \mathrm{~L} / \mathrm{s}(6 \mathrm{~L} / \mathrm{min})$ de agua en $0.5 \mathrm{~m}^{2}$ de tela con un simulador de lluvia y se mide en 30 minutos cuanto volumen agua se perdió al infiltrarse por las telas recubiertas y que no fue colectada en los medidores pluviométricos que van conectados a estas. Unidades $\mathrm{L} / \mathrm{m}^{2}$.

Todas las pruebas de lluvia fueron realizadas con un simulador de lluvia marca Armfield con escala de 0.01 a $100 \mathrm{~L} / \mathrm{s}$. A partir de los datos obtenidos con estos estudios, se procedió a realizar el diseño de los filtros empleados en los sistemas y que van sostenidos en mangueras flexibles o "lianas" como se les han denominado, en donde se realiza el proceso de filtración y la descarga del agua a un tanque con sistemade tratamiento germicida. Esto último con el fin de alcanzar una calidad de agua para consumo humano conforme a los parámetros de la NOM-127-SSA1-1994.

\section{Resultados y discusión.}

\section{Estudio de la calidad del agua de lluvia colectada}

Resultados de los estudios de la calidad del agua de las cuatro delegaciones monitoreadas.

Tabla 2. Características microbiológicas del agua de lluvia en el Distrito Federal .

\begin{tabular}{ccccc}
\hline \multirow{2}{*}{ Parámetro } & $\begin{array}{c}\text { Límite } \\
\text { permisible }\end{array}$ & Tlalpan & $\begin{array}{c}\text { Miguel } \\
\text { Hidalgo }\end{array}$ & Coyoacán \\
\cline { 3 - 5 } & & 6 & 4 & 3.875 \\
\hline Organismos & 2 & $\mathrm{UFC} / 100$ & $\mathrm{UFC} / 100$ & $\mathrm{UFC} / 100$ \\
coliformes & $\mathrm{UFC} / 100 \mathrm{ml}$ & $\mathrm{ml}$ & $\mathrm{ml}$ & $\mathrm{ml}$ \\
totales & & 2 & & 1.8571 \\
$\begin{array}{c}\text { Organismos } \\
\text { coliformes } \\
\text { fecales }\end{array}$ & $\mathrm{UFC} / 100 \mathrm{ml}$ & $\mathrm{UFC} / 100$ & $\mathrm{ND}$ & $\mathrm{UFC} / 100$ \\
& & $\mathrm{ml}$ & & $\mathrm{ml}$ \\
\hline
\end{tabular}

Tabla 3. Características fisicoquímicas del agua pluvial en el Distrito Federal.

\begin{tabular}{|c|c|c|c|c|}
\hline Parámetro & $\begin{array}{c}\text { Límite } \\
\text { permisible } \\
(\mathrm{mg} / \mathrm{l})\end{array}$ & $\begin{array}{c}\text { Tlalpan } \\
\text { (mg/l) }\end{array}$ & $\begin{array}{c}\text { Miguel } \\
\text { Hidalgo } \\
\text { (mg/l) }\end{array}$ & $\begin{array}{c}\text { Coyoacán } \\
\text { (mg/l) }\end{array}$ \\
\hline $\begin{array}{c}\text { Cloro } \\
\text { residual libre }\end{array}$ & $0.2-1.50$ & 0.9 & 1.35 & 0.7625 \\
\hline $\begin{array}{l}\text { Cloruros } \\
\left(\text { como } \mathrm{Cl}^{-}\right)\end{array}$ & 250 & 127 & 202 & 20.125 \\
\hline $\begin{array}{c}\text { Dureza total } \\
\text { (como } \\
\left.\mathrm{CaCO}_{3}\right)\end{array}$ & 500 & 268.5 & 357.5 & 170.625 \\
\hline $\begin{array}{l}\text { Fluoruros } \\
\text { (como F-) }\end{array}$ & 1.5 & 0.25 & 0.5 & 0.9875 \\
\hline $\begin{array}{l}\text { Nitratos } \\
(\text { como N) }\end{array}$ & 10 & 1.5 & 2.5 & 3.5 \\
\hline $\begin{array}{l}\text { Nitritos } \\
\text { (como N) }\end{array}$ & 0.05 & ND & ND & 0.0255 \\
\hline $\begin{array}{l}\text { Nitrógeno } \\
\text { amoniacal }\end{array}$ & 0.5 & 0.4 & 0.1 & 0.3575 \\
\hline $\begin{array}{c}\mathrm{pH} \\
\text { (potencial de } \\
\text { hidrógeno) }\end{array}$ & $6.5-8.5$ & 3 & 3.8 & 5.625 \\
\hline $\begin{array}{l}\text { Sólidos } \\
\text { disueltos } \\
\text { totales }\end{array}$ & 1000 & 100 & 200 & 218.5 \\
\hline $\begin{array}{c}\text { Sulfatos } \\
\left(\text { como } \mathrm{SO}_{4}{ }^{2-}\right)\end{array}$ & 400 & 55 & 75 & 192.625 \\
\hline $\begin{array}{c}\text { Sustancias } \\
\text { activas al } \\
\text { azul de } \\
\text { metileno }\end{array}$ & 0.5 & 0.1 & 0.1 & 0.375 \\
\hline
\end{tabular}

Tabla 4. Contaminantes orgánicos persistentes encontrados en el agua de lluvia de las delegaciones.

\begin{tabular}{|c|c|c|c|c|}
\hline Parámetro & $\begin{array}{c}\text { Límite } \\
\text { permisible } \\
(\mathrm{mg} / \mathrm{l})\end{array}$ & $\begin{array}{c}\text { Tlalpan } \\
\text { (mg/l) }\end{array}$ & $\begin{array}{c}\text { Miguel } \\
\text { Hidalgo } \\
\text { (mg/l) }\end{array}$ & $\begin{array}{c}\text { Coyoacán } \\
\text { (mg/l) }\end{array}$ \\
\hline $2,4-\mathrm{D}$ & 50 & 4.5 & 10 & 1.8125 \\
\hline $\begin{array}{l}\text { Clordano } \\
\text { (total de } \\
\text { isómeros) }\end{array}$ & 0.3 & 0.05 & 0.1 & 0 \\
\hline $\begin{array}{c}\text { DDT } \\
\text { (total de } \\
\text { isómeros) }\end{array}$ & 1 & nd & nd & 0.115 \\
\hline Fenoles & 0.001 & nd & nd & 0.001 \\
\hline $\begin{array}{c}\text { Gamma-HCH } \\
\text { (lindano) }\end{array}$ & 2 & nd & 1.5 & 0.05 \\
\hline $\begin{array}{l}\text { Hexacloro- } \\
\text { benceno }\end{array}$ & 0.01 & 0.005 & 0.02 & 0 \\
\hline Heptacloro & 0.03 & 0.01 & 0.035 & 0 \\
\hline Metoxicloro & 20 & 2.5 & 10 & 2.625 \\
\hline $\begin{array}{l}\text { Plaguicidas } \\
\text { totales }\end{array}$ & 0.03 & 0.015 & 0.022 & 0.01 \\
\hline $\begin{array}{l}\text { Trihalo- } \\
\text { metanos }\end{array}$ & 0.2 & 0.24 & 0.5 & 0 \\
\hline
\end{tabular}




\section{Telas recubiertas}

Las pruebas de las telas recubiertas dieron los siguientes resultados:

Tabla 5. Infiltración estática de mallas tratadas. Unidades $\mathrm{L} / \mathrm{m}^{2} \cdot \min$

\begin{tabular}{ccccc}
\hline $\begin{array}{c}\text { Material } \\
\text { de malla }\end{array}$ & $\begin{array}{c}\text { Polímero } \\
\text { hidrófobo }\end{array}$ & $\begin{array}{c}\text { Cera } \\
\text { carnauba }\end{array}$ & $\begin{array}{c}\text { Cera } \\
\text { abeja }\end{array}$ & $\begin{array}{c}\text { Cera } \\
\text { sintética } \\
\text { (ceramida) }\end{array}$ \\
\hline $\begin{array}{c}\text { Polímero } \\
\text { A }\end{array}$ & 0.042 & 0.002 & 00 & 0.005 \\
$\begin{array}{c}\text { Polímero } \\
\text { B }\end{array}$ & 0.081 & 0.003 & 0.001 & 0.007 \\
Tela A & 0.125 & 0.004 & 0.001 & 0.02 \\
Tela B & 0.056 & 0.002 & 00 & 0.009 \\
\hline
\end{tabular}

Tabla 6. Infiltración dinámica de mallas tratadas. Unidades $\mathrm{L} / \mathrm{m}^{2}$

\begin{tabular}{ccccc}
\hline $\begin{array}{c}\text { Material } \\
\text { de malla }\end{array}$ & $\begin{array}{c}\text { Polímero } \\
\text { hidrófobo }\end{array}$ & $\begin{array}{c}\text { Cera } \\
\text { carnauba }\end{array}$ & $\begin{array}{c}\text { Cera } \\
\text { abeja }\end{array}$ & $\begin{array}{c}\text { Cera } \\
\text { sintetica } \\
\text { (ceramida) }\end{array}$ \\
\hline $\begin{array}{c}\text { Polímero } \\
\text { A }\end{array}$ & 0.54 & 0.17 & 0.06 & 0.18 \\
$\begin{array}{c}\text { Polímero } \\
\text { B }\end{array}$ & 0.63 & 0.31 & 0.04 & 0.22 \\
Tela A & 0.77 & 0.23 & 0.01 & 0.15 \\
Tela B & 0.64 & 0.21 & 0.02 & 0.14 \\
\hline
\end{tabular}

Estos resultados indican que las ceras de abeja y carnauba son las más adecuadas para usarse como recubrimiento hidrofóbico de las mallas pero en forma estática, porque al realizar la prueba dinámica solo la cera de abeja se mantiene impermeable y el recubrimiento con cera carnauba al parecer es arrastrado por el flujo de agua permitiendo una humectación mayor (menor ángulo de contacto)

Los ángulos de contacto se obtuvieron de los experimentos con los goniómetros y los coeficientes de se calcularon con la Ecuación 2.

Tabla 7. Ángulos de contacto $(\theta)$ para los recubrimientos de las telas. Liquido empleado agua bidestilada.

\begin{tabular}{ccccc}
\hline $\begin{array}{c}\text { Material } \\
\text { de malla }\end{array}$ & $\begin{array}{c}\text { Polímero } \\
\text { hidrófobo } \\
\text { de silicón }\end{array}$ & $\begin{array}{c}\text { Cera } \\
\text { carnauba }\end{array}$ & $\begin{array}{c}\text { Cera } \\
\text { abeja }\end{array}$ & $\begin{array}{c}\text { Cera } \\
\text { sintética } \\
\text { (ceramida) }\end{array}$ \\
\hline Polímero A & 86.5 & 95.3 & 110.5 & 101.2 \\
Polímero B & 82.8 & 91.4 & 104.6 & 96.4 \\
Tela A & 75.7 & 88.4 & 93.7 & 92.2 \\
Tela B & 72.1 & 86.2 & 96.4 & 94.7 \\
\hline
\end{tabular}

Tabla 8. Coeficientes de cohesión calculados a partir de los ángulos de contacto $(\theta)$ para los recubrimientos de las telas. Liquido empleado agua bidestilada.

\begin{tabular}{ccccc}
\hline $\begin{array}{c}\text { Material } \\
\text { de malla }\end{array}$ & $\begin{array}{c}\text { Polímero } \\
\text { hidrófobo } \\
\text { de silicón }\end{array}$ & $\begin{array}{c}\text { Cera } \\
\text { carnauba }\end{array}$ & $\begin{array}{c}\text { Cera } \\
\text { abeja }\end{array}$ & $\begin{array}{c}\text { Cera } \\
\text { sintética } \\
\text { (ceramida) }\end{array}$ \\
\hline $\begin{array}{c}\text { Polímero } \\
\text { A }\end{array}$ & 0.48 & 0.52 & 0.61 & 0.56 \\
Polímero B & 0.46 & 0.50 & 0.6 & 0.53 \\
Tela A & 0.42 & 0.48 & 0.52 & 0.51 \\
Tela B & 0.4 & 0.47 & 0.53 & 0.52 \\
\hline
\end{tabular}

La cera de abeja presenta el mayor ángulo de contacto para el agua por tanto la energía de superficie del solido (tela recubierta) es menor. Esto implica mayores energías en la tensión superficial del líquido y por tanto mayor cohesión. De ahí que este tipo de cera sea el recubrimiento más impermeable.

\section{Captadores diseñados}

La figura 7 presenta el primer prototipo, que consta de una malla tipo "tela de araña" que se encuentra en tensión mediante postes desmontables y por lo tanto lo hacen de fácil armado, el presente material esta hecho de tela de algodón reforzada con polietileno con recubrimiento de ceras naturales o ceramidas.

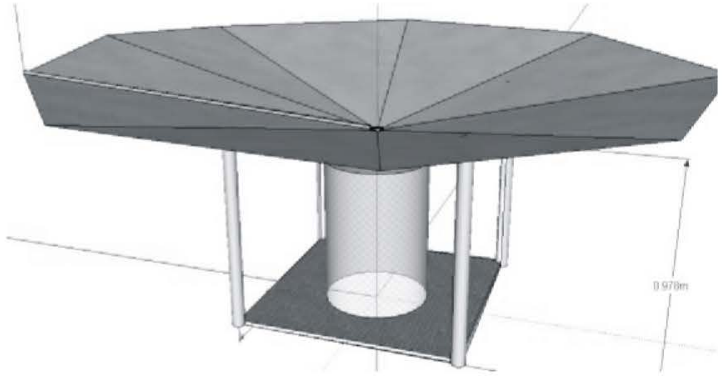

Figura 7. Prototipo de captador tela de araña.

Los postes y la estructura son de estireno y PVC. Consta de 4 elementos;

1. La tela de araña reforzada (área de captación).

2. Estructura y tubería de PVC.

3. Tanque de almacenamiento de acrílico (solo en modelo sin liana).

4. Filtros 


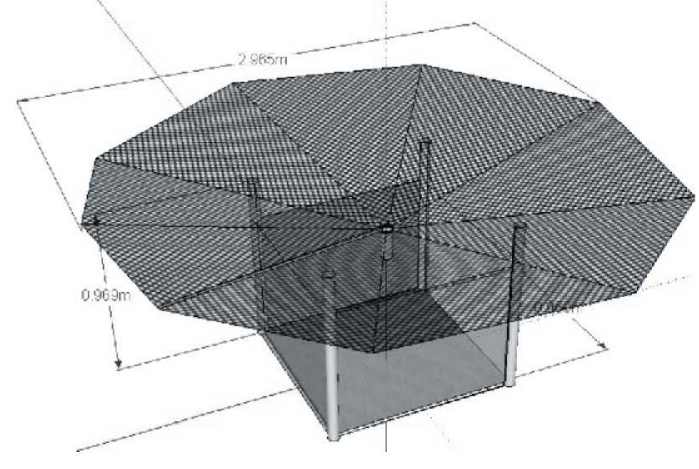

Figura 8. Segundo prototipo tipo tela de araña.

En base a la estructura del prototipo anterior se realizó una modificación en la "tela de araña" reemplazándola por estireno como nuevo medio de captación.

\section{Diseño de filtros y prototipos y su estudio a nivel planta piloto}

Para la primera fase de filtración, los filtros se encuentran constituidos de piedra de río, arena, y arcilla mientras en la segunda fase se ocupa un filtro a base de carbón activado y otro biológico, cuya finalidad es adsorber contaminantes de tipo orgánico.

\section{Filtro de zeolita/grava}

La tubería hidráulica del agua filtrada consiste de un tubo de PVC hidráulico de 4" de diámetro, para la captación del agua filtrada y la parte que sale fuera del recipiente por la parte inferior empotrada con un cople y una reducción de 4 a $1 / 2 "$. El material filtrante, consiste de una capa de $10 \mathrm{~cm}$. de zeolita (de $0.5 \mathrm{~cm}$ de diámetro), colocada en el fondo del recipiente, envolviendo la tubería hidráulica y sobre ella una capa de $28 \mathrm{~cm}$. de zeolita, de tamaño efectivo entre 3 y $4 \mathrm{~mm}$., con coeficiente de uniformidad menor que 5 .

El plato difusor tendrá perforaciones de $1 / 4$ " de pulgada, distribuidas uniformemente en todo el plato. Este dispositivo tiene la finalidad de recibir el agua sin filtrar y distribuirla uniformemente para evitar los disturbios que se podrían provocar sobre la capa de arena si se descargara el agua directamente sobre ella.

\section{Especificaciones técnicas del diseño.}

Para el diseño se adaptó un tubo de PVC hidráulico de diámetro de 4". En su interior tiene un lecho filtrante compuesto por dos capas: una inferior de $0.05 \mathrm{~mm}$ de gravilla (1/4 a $1 / 2 ")$, y una superior de $0.30 \mathrm{~m}$ de arena gruesa $(2-6 \mathrm{~mm})$. Interiormente se coloca una simple tubería en PVC de $1 / 2$ " para captar las aguas filtradas que salen por la parte superior por simple derrame. El filtro se basa en una velocidad de filtración de $60 \mathrm{~m}^{3} / \mathrm{m}^{2} \mathrm{x} \mathrm{h}$.

Debido a que se tiene una tubería hidráulica de $4 "$, esto equivale a aproximadamente $10 \mathrm{~cm}$, entonces para calcular el flujo filtrado con estas características se aplica la siguiente ecuación:

$$
\begin{gathered}
\text { Area de la tuberia: } \pi r^{2}=\pi \cdot 5:=78.54 \mathrm{~cm}: \\
60 \frac{\mathrm{m}^{3}}{\mathrm{~m}^{2} \cdot \mathrm{hr}} \cdot|78.54 \mathrm{~cm}:| \cdot\left|\frac{1 \mathrm{~m}^{*}}{(100 \mathrm{~cm})^{2}}\right| \cdot\left|\frac{1000 \mathrm{~L}}{1 \mathrm{~m}^{2}}\right|=471.24 \frac{\mathrm{l}}{\mathrm{hr}}=7.854 \frac{\mathrm{l}}{\mathrm{min}}
\end{gathered}
$$

El filtro opera con porciones de 5 litros por vez. El agua se verterá por la parte superior ese volumen y colocar el recipiente de forma de recibir el agua filtrada por la boca de descarga

En el tratamiento de aguas residuales, la filtración es una operación utilizada para remover sólidos, material no sedimentables, turbiedad, fósforo, $\mathrm{DBO}, \mathrm{DQO}$, metales pesados, virus; para asegurar una calidad superior del efluente secundario. La filtración se puede usar para depurar efluentes secundarios, sin agregar coagulantes; con agresión de coagulantes, antes de la filtración, o antes del sedimentador secundario y para depurar aguas residuales crudas previamente coaguladas, floculadas y sedimentas, en una planta de tratamiento físico-químico.

En la Figura 9 podemos observar los diferentes tipos de filtros típicos de arena que existen para el tratamiento de agua.

La finalización de la carrera de filtración se determina por la fuga de sólidos en el efluente filtrado (turbiedad superior al límite aceptable), o porque la pérdida de energía, a través del filtro, alcanza el valor máximo permisible; finalizada esta carrera, hay que lavar el filtro para remover los sólidos retenidos y para recuperar la capacidad de filtración. Tomando en cuenta que este filtro biológico es de tipo no estratificado, se utilizará un retrolavado con aire y agua. En algunos estudios se indica que la frecuencia de limpieza de los filtros lentos varía de una a dos veces por mes, con remoción del $60 \%$ de SS y de $40 \%$ de DBO [12]. 


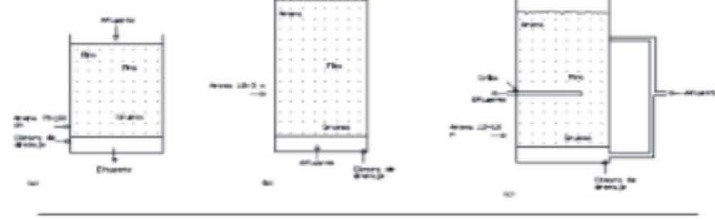

Figura 9. Tipos de Filtros: (a) Filtro convencional de arena; (b) Filtro ascensional de arena; (c) Filtro de arena de flujo dual; (e) Filtro de Lecho mezclado. (Romero, 2008).

\section{Criterios de diseño}

La diferencia principal entre los filtros para purificación de agua y los filtros para tratamiento de aguas residuales radica en el tamaño del medio filtrante. Los granos del medio filtrante para aguas residuales deben ser más grandes para que el filtro tenga una velocidad apropiada de filtración y pueda almacenar el volumen de flóculo removido, parar el diseño de este filtro se tomó como base los criterios de diseño similares al filtro de arena típico, ya que no existe una manera exacta de diseñar filtros empacados con material biológico. Otras dos variables importantes en filtración de aguas residuales son la resistencia del floculo y la concentración de sólidos suspendidos, en este caso, los metales pesados. El diseño de un filtro para aguas residuales requiere una selección apropiada del tamaño del medio filtrante, de la profundidad del lecho de filtración, de la tasa de filtración y de la pérdida de carga disponible para filtración. Evidentemente, la mejor manera de seleccionar dichos parámetros y proveer un diseño de costos mínimos es desarrollar un estudio con planta piloto que permita deducir los requisitos del sistema de filtración, con el cual, en este proyecto se pretende diseñar el sistema de tratamiento para su posterior construcción y puesta en marcha. En plantas de filtración de aguas residuales es necesario evaluar los siguientes factores:

a) Efectos potenciales de caudales pico y de variaciones continuas de caudal entre período de tiempo seco y de tiempo lluvioso que pueden hacer necesario proveer un tanque de igualamiento de mezcla completa antes de los filtros.

b) En períodos de caudales pico las cargas de sólidos suspendidos (SS) pueden alcanzar valores tan altos como 30 a $50 \mathrm{mg} / \mathrm{L}$ de sólidos suspendidos y turbiedades mayores de 15 UNT, lo cual contribuye a pérdidas de carga altas y carreras de filtración cortas. Para contrarrestar este efecto se puede utilizar un lecho más grueso, o un lecho dual o mezclado que permita filtración gruesa o fina, así como una altura mayor de energía para filtración o recurrir a filtros de presión. Por fines económicos, este tratamiento en general se basa en la gravedad, no se trabajó con presión.

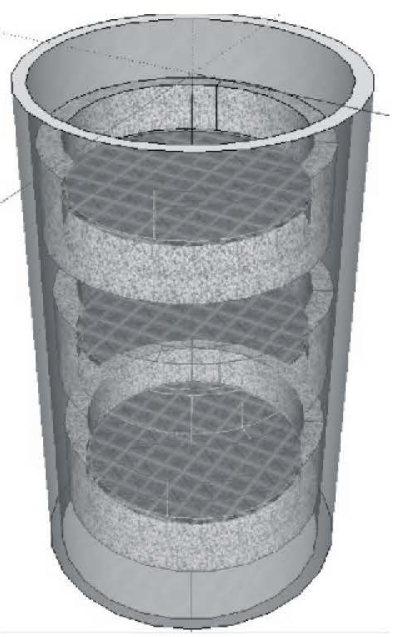

Figura 10. Imagen del prototipo de los filtros. Pruebas de materiales adsorbentes y su relleno en tubos flexibles para lianas colectoras.

La siguiente parte de este proyecto está conformada por un prototipo denominado liana el cual se presenta a continuación:

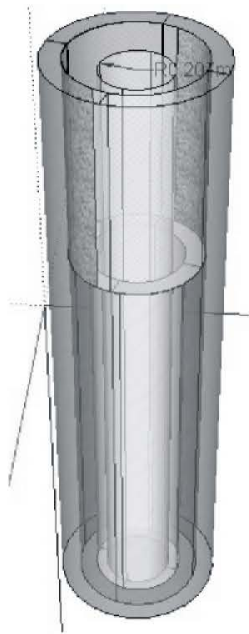

Figura 11. Imagen de prototipo de lianas colectoras.

Este sistema representado en la imagen anterior posee un doble tubo, en donde, el tubo interior se encuentra enchaquetado, y en la parte exterior e interior de esta está representado el material que le 
confiere la elasticidad, para que pueda ser una liana articulada.

\section{Diseño de prototipos}

El diseño final del captador pluvial se realizó mediante el método de la fórmula racional Aquí se presentan los resultados de pruebas de captación una vez construido y probado en un simulador de lluvia Armfield.

El cálculo del gasto racional, como se indica a continuación.

$$
Q P=2.778 C I A
$$

\section{Donde:}

$\mathrm{Qp}=$ Gasto pluvial, en $1 / \mathrm{s}$

$\mathrm{A}=$ Área de captación, en hectáreas.

$\mathrm{C}=$ Coeficiente de escurrimiento, adimensional

$\mathrm{I}=$ Intensidad de precipitación, en $\mathrm{mm} / \mathrm{hr}$.

El coeficiente de escurrimiento se tomó de las normas técnicas complementarias para el diseño y ejecución de obras e instalaciones hidráulicas.

$$
\begin{aligned}
& \mathrm{A}=6640 \mathrm{~cm}^{2}=0.664 \mathrm{~m}^{2}=0.0000664 \mathrm{ha} \\
& \mathrm{C}=0.70 \\
& \mathrm{I}=5400 \mathrm{~mm} / \mathrm{hr} \\
& \mathrm{Qp}=2.778(0.0000664 \mathrm{ha})(0.70)(5400 \mathrm{~mm} / \mathrm{hr})= \\
& 0.6972 \mathrm{~L} / \mathrm{s}
\end{aligned}
$$

Con el dato anterior se concluye que el equipo capto 5.4 litros de 41.83 litros de precipitación en un minuto.

La calidad del agua obtenida de este captador cumple con un $80 \%$ de los parámetros que marca la NOM 127 pero son los coliformes fecales y totales, así como algunos tóxicos recalcitrantes (hidrocarburos y plaguicidas) los que aún persisten en trazas en el agua pluvial después de la filtración, lo que obliga a adaptar al sistema un filtro foto catalítico y germicida como son los de procesos oxidativos avanzados (POA) de óxido de titanio estimulados con luz UV.

\section{Conclusiones}

1. Se ha logrado diseñar un primer prototipo, que opera con una presión máxima de $5 \mathrm{~kg} / \mathrm{m}^{2}$ y un flux de $14 \mathrm{~m}^{3} / \mathrm{m}^{2} \mathrm{xh}$ pero con obturación después de 3 horas de trabajo. La calidad del agua obtenida se acerca a la NOM-127-SSA.

2. Cada año el aporte de agua de lluvia en el DF es de casi 780 millones de $\mathrm{m}^{3}$ pero solo cerca del $13 \%$ de este volumen, se aprovecha. Con los captadores-filtro podemos lograr por área de cobertura para el D.F. tener casi 2 meses de abastecimiento autónomo (sin consumo de la red) y alcanzar un aprovechamiento superior al $40 \%$ del aporte de agua de lluvia.

\section{Agradecimientos}

Los autores agradecen al Instituto de Ciencia y Tecnología del Distrito Federal (ICYTDF) por el financiamiento para realizar el presente trabajo, a través del proyecto ICYTDF-IPN 0246.

\section{Referencias}

1. Gleason, A. Manual de Aprovechamiento de aguas pluviales en centros urbanos. Universidad de Guadalajara. 2005;15-16

2. Capella, A. Agua, Usos, Abusos, Problemas y Soluciones. VII. La Cuenca del Valle de México. La Problemática Hidráulica del Valle de México: Edit. Mapas, 2006; pp. 229234.

3. http://www.todoagua.aqua-soft.com.mx/filtros-filtracionpurificadores-para-agua-filtros-en-linea-c-25_58.html

4. 4De La Macorra, C. Agua, Usos, Abusos, Problemas y Soluciones. VIII. La Gobernabilidad del Agua. El Caso Monterrey. Historia de un Pionero. Edit. Mapas, 2006; pp. 241-258.

5. http://smn.cna.gob.mx/

6. http://www.inegi.org.mx/

7. http://www.conafor.gob.mx

8. Soto, A. Diagnostico sobre la situación de riesgo y vulnerabilidad de los habitantes del distrito federal al no contar con el servicio de agua potable, como base para el análisis del derecho humano al agua y los derechos colectivos de los habitantes. 2000.

9. Lara, L. Informe climatológico ambiental del valle de México. 2006.

10. Palacio., F. Propuesta de un sistema de aprovechamiento de agua lluvia, como alternativa para el ahorro de agua potable. 2010.

11. Nom-127-SSA1-1994

12. Romero, L. 2008. Diseño de filtros biológicos IPN México, D.F. p.32-44. 